\title{
Food Science Dialog 2015
}

\author{
Jan Fritsche ${ }^{1} \cdot$ Martin Holle $^{1} \cdot$ Katharina Riehn ${ }^{1}$. \\ Gregor Buschhausen-Denker ${ }^{2} \cdot$ Till Sager $^{2} \cdot$ Antje Steinbicker $^{2}$
}

Published online: 17 October 2015

(C) Bundesamt für Verbraucherschutz und Lebensmittelsicherheit (BVL) 2015

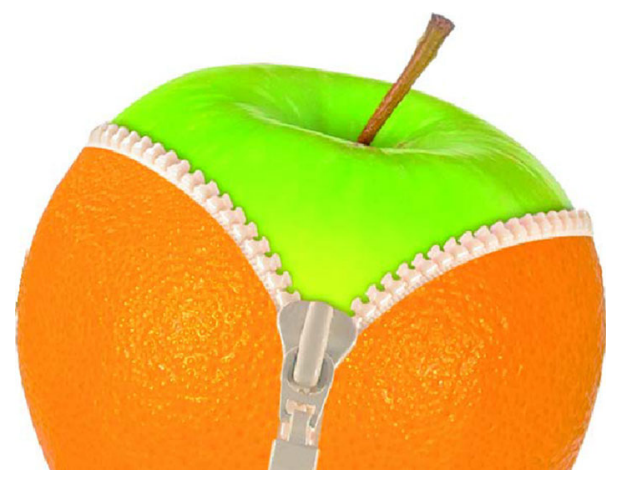

Der Schutz der Verbraucher vor Irreführung bei Lebensmitteln ist schon immer ein zentrales Anliegen von Lebensmittelrecht und Lebensmittelüberwachung gewesen. Mit der zunehmenden Globalisierung der Lebensmittelkette einerseits und dem Trend zur Auslobung regional und lokal erzeugter Lebensmittel andererseits entstehen neue Gefährdungslagen im Hinblick auf eine potentielle Verbrauchertäuschung. Die Bedingungen für die Auslobung einer regionalen oder besonderen geografischen Herkunft sind deshalb klar zu definieren und für die Lebensmittelüberwachung überprüfbar zu machen. Das stellt sowohl den Rechtsanwender als auch die Wissenschaft vor Herausforderungen. Gleichzeitig nimmt mit zunehmender Bereitschaft

Jan Fritsche

Jan.Fritsche@haw-hamburg.de

1 Hochschule für Angewandte Wissenschaften (HAW), Hamburg, Germany

2 Behörde für Gesundheit und Verbraucherschutz (BGV), Hamburg, Germany der Verbraucher, für regionale Spezialitäten einen höheren Preis zu zahlen, das Risiko betrügerischer Praktiken zu. Dem kann nur mit Analysemethoden begegnet werden, die trennscharf Produkte voneinander unterscheiden können, die nur in wenigen Merkmalen voneinander abweichen.

Der jährlich stattfindende Food Science Dialog in Hamburg wurde von der HAW Hamburg, der Behörde für Gesundheit und Verbraucherschutz (BGV) und der Behörde für Wissenschaft und Forschung (BWF) und in Kooperation mit der Universität Wageningen, Niederlande, organisiert und durchgeführt. Er ist sowohl von der Akademie für Tierärztliche Fortbildung als auch von der Zertifizierungsstelle für die Fortbildung von Lebensmittelchemikern anerkannt und bietet ein Forum, um sich Fragestellungen rund um Lebensmittel aus dem Blickwinkel verschiedener fachlicher Disziplinen zu nähern. Experten aus Wissenschaft, Behörden, Rechtsberatung und Unternehmen der Lebensmittelbranche diskutierten aktuelle Themen, unterstützt und unter aktiver Beteiligung von Hamburger Lebensmittelkontrolleuren, Studierenden der Studiengänge Food Science und Ökotrophologie sowie von Vertretern der regionalen Lebensmittelwirtschaft.

Unter dem Titel „Lebensmittel im Fokus: Schutz vor Irreführung - regional, global und online“ stand vom 14. bis 18. September 2015 eine abwechslungsreiche Mischung aus nationalen und internationalen Fachvorträgen, eine Exkursion zur Containerprüfanstalt Hamburg sowie Verkostungen von Olivenöl, Kaviar und Honig auf dem Programm, um eine enge Verzahnung von Theorie und Praxis zu schaffen. Ein besonderer Schwerpunkt lag dabei auch auf den 
analytischen Methoden zur Überprüfung der Authentizität von Lebensmitteln. Außerdem wurden die rechtlichen Rahmenbedingungen für die Vermarktung regionaler Erzeugnisse dargestellt, und neue Wege der Kontrolle des Handels im Internet präsentiert (Lebensmittelkontrolle 2.0). Auch die aktuellen Herausforderungen des globalen Handels (TTIP) wurden ausführlich diskutiert.

Das vorliegende Heft fasst die Ergebnisse der fünftägigen Fortbildungsveranstaltung zusammen. 